Journal Homepage: -www.journalijar.com
ISSN NO. 2320-5407

RESEARCH ARTICLE

\title{
BIODIVERSITY STUDY OF LAKE SHORELINES OF BENGALURU PERI URBAN AREA AND ITS MANAGEMENT.
}

\author{
Bheemappa $\mathrm{K}$ and Nandini $\mathbf{N}$ \\ Professor and Research Scholar, Department of Environmental Science, Bangalore University, Bengaluru 560056, \\ Karnataka, India.
}

\section{Manuscript Info}

Manuscript History

Received: 22 July 2018

Final Accepted: 28 August 2018

Published: September 2018

Keywords:-

Shoreline, Biodiversity, Management,

Sustainable, Bengaluru.

\section{Abstract}

Lakes are the most productive and biologically rich endangered ecosystems and they function as an interface between land and water systems and its Biodiversity severely are threatened globally by inappropriate management measures taken for protection of Lake Shorelines. To bear out the point of the Biodiversity Study of Lake Shoreline of Bengaluru peri urban area and its management, 7 lakes namely Dorekere lake, Hosakerehalli lake and Subramanyapura lake, Margondanahalli Hosakere lake, Kommaghatta lake, Bandematta lake and Byramangala lake were selected for sampling in the peri-urban area of Vrishabhavathi river basin in Byramangala series. Biodiversity Study for the Identification of Flora and Fauna in the Shoreline of Studied lakes were carried out by following the methods of William (2006), Subramanian, (2005; 2009), Mark et al.,(2000), Richard et al.,(2002), Krushnamegh Kunte (2008),Jayaram, (1999),Rema and Indra (2009), Hutchins et al., (2003); Nelson, (2006); Quentin Richard and Moore (2008), Fredrick et al., (2011), Bookhout (1994), Donald and Richard (1970), MeenakshiVenkataraman (2010), Subramanian and Sivaramakrishnan (2007) and Karen Edelstein (1999). The overall recorded number of species of Flora and Fauna in the study area were recorded between 146 species to 171 species. They were found to be highest in number of species presence is due to recent rejuvenation and plantation programmes carried out in the studied lakes, the local associations and citizens have also adopted the plants for the maintenance and protection, are in the less populated zone, and are situated away from the village and near to reserve forest cover. The less no of species were found due to the lakes situated in urbanised and industrialised area.

Copy Right, IJAR, 2018,. All rights reserved.

\section{Introduction:-}

The environmental condition of any Lake system depends upon the nature of that lakes and its exposure to various ecological factors. These fragile ecosystems must maintain the state of environmental equilibrium with the existing condition of the surroundings. Lakes are the most productive and biologically rich endangered ecosystems and they function as an interface between land and water systems. They filter sediments and nutrients from surface water and support all life forms through extensive food webs and biodiversity such as critical habitat for plants and animals 
together with bacteria, fungi, algae, plankton, mussels, snails, crustaceans, insects, fish, amphibians, reptiles, birds and mammals. These types of Lake Biodiversity severely are threatened globally by inappropriate management measures taken for protection of Lake Shorelines.

\section{Materials And Methods:- Description of Study Area:}

To bear out the point of the Biodiversity Study of Lake Shoreline of Bengaluru peri urban area and its management, 7 lakes namely Dorekere lake, Hosakerehalli lake and Subramanyapura lake, Margondanahalli Hosakere lake, Kommaghatta lake, Bandematta lake and Byramangala lake were selected for sampling in the peri-urban area of Vrishabhavathi river basin in Byramangala series.

Table 1:-Coordinates indicating the lakes in the study area

\begin{tabular}{|l|c|c|}
\hline Name of the lake & Latitude & Longitude \\
\hline Margondanahalli Hosakere & $12^{\circ} 56^{\prime} 07.98^{\prime \prime} \mathrm{N}$ & $77^{\circ} 26^{\prime} 01.43^{\prime \prime} \mathrm{E}$ \\
\hline Kommaghatta & $12^{\circ} 55^{\prime} 49.12^{\prime \prime N}$ & $77^{\circ} 27^{\prime} 58.69^{\prime \prime} \mathrm{E}$ \\
\hline Bandematta & $12^{\circ} 55^{\prime} 28.25^{\prime \prime} \mathrm{N}$ & $77^{\circ} 28^{\prime} 51.75^{\prime \prime} \mathrm{E}$ \\
\hline Hosakerehalli & $12^{\circ} 92^{\prime} 54.36^{\prime \prime N}$ & $77^{\circ} 53^{\prime} 39.93^{\prime \prime} \mathrm{E}$ \\
\hline Dorekere & $12^{\circ} 90^{\prime} 29.04^{\prime \prime N}$ & $77^{\circ} 51^{\prime} 10.67^{\prime \prime} \mathrm{E}$ \\
\hline Subramanyapura & $12^{\circ} 89^{\prime} 68.74^{\prime \prime N}$ & $77^{\circ} 54^{\prime} 24.69^{\prime \prime} \mathrm{E}$ \\
\hline Byramangala & $12^{\circ} 75^{\prime} 95.42^{\prime \prime N}$ & $77^{\circ} 41^{\prime} 85.16^{\prime \prime} \mathrm{E}$ \\
\hline
\end{tabular}

The following are the methods followed for the Identification of Flora and Fauna in the Shoreline of Studied lakes and tabulated in terms of presence or absence;

Trees in the $50 \mathrm{~m}$ vicinity of water-bodies were individually counted to assessing the densities of large trees (William, 2006).

Shrubs and Herbs in the vicinity of water-bodies were quantified by using the intensive quadrant. The quadrant was in square shape, which adapted to characterise the floral community. For the herbaceous plants $1 \mathrm{~m} x 1 \mathrm{~m}$ quadrant was made (William, 2006).

Aquatic plants include floating plants, submerged and rooted plants which were quantified by using $1 \mathrm{~m} \times 1 \mathrm{~m}$ quadrat wooden frame, which is used to sample floating or emergent vegetation on the water surface (William, 2006). To standardize the study, 5 quadrats were laid down in the water-body having dense aquatic vegetation.

Odonates sample was conducted for adult dragonfly and damselfly of the water-bodies from the time of 10.00 to 14.00 (midday), where the most Odonate are active by walking along the offshore where ever possible and if the water-body is large, then a portion of water-body shore was considered. As a rule, dragonflies and damselflies were identified (Subramanian, 2005; 2009) by sight for 30 minutes and occasionally the Odonates was captured by using nets when there was doubt in identification.

Bird sampling strategy was based on 'random stratified sampling' (Mark et al., 2000). Sampling points are randomly located throughout the water-body area to obtain representative samples of the species and numbers of each species. 'Point counts' methodology was followed for essentially strip transects of zero length, in which the count is $360^{\circ}$ arcs around a fixed sampling point. The radius of $50 \mathrm{~m}$ was used and the birds were counted for 10 minutes. Birds that are seen flying over the census area (aerial species) are recorded separately because they cannot be included in standard density estimation. The birds were identified using Binoculars and field guide (Richard et al., 2002).

Butterflies in the water-bodies were sampled by 'searching and direct observation'. The method was adopted especially for productive in fairly open habitats with sparse vegetation; direct observation is clearly the basis of recording day flying butterflies. The sampling was done for 1 hour in the water-body area and repeated for 10 times in a season on fine days (William, 2006). Butterflies were identified by use the field manual by Krushnamegh Kunte (2008). 
Fishes found in the water-bodies were identified by collecting the samples by using the experimental multifilament gillnets of varying mesh size from $8 \mathrm{~mm}$. Two fleets of gillnet were set one inshore and another offshore (about 100 $\mathrm{m}$ from shoreline). The retrieved fish specimens were sorted; structure determined and identified using external morphological characteristics and identification keys (Jayaram, 1999; Rema and Indra 2009; Hutchins et al., 2003; Nelson, 2006; Quentin Richard and Moore, 2008). Collected fishes were counted on species basis and total numbers of each species caught per study site recorded in a catch composition data sheet (Fredricket al., 2011).

Mammals, which found in the water-body areas as migration form other than human were evaluated by 'Total counts' (Bookhout, 1994) and 'Holy Grail of mammal census method'.

Insects in and near the water-body was collected by using the 'Sweep net', which was swung through the vegetation to dislodge the specimens. Each sample consists of 10 sweeps considering the area from ground level to 2 meter (Janzen, 1968). Sweep net has $1.5 \mathrm{~mm}$ mesh size with $30 \mathrm{~cm}$ diameter and the collected species been identified by followed the manuals of Donald and Richard (1970) and Meenakshi Venkataraman (2010). Aquatic insects was carried out by taking a fixed number of sweeps, netting for a set period (typically up to 3 minutes, during which time the net may need emptying as it becomes clogged with vegetation) or continuing to samples and sort the catch for a set period (typically 30-45 min)(William, 2006). The aquatic insects were identified by following the field manual by Subramanian and Sivaramakrishnan (2007) and Karen Edelstein (1999). All the biodiversity carried out once for the research study during the years between 2012-2015. However, during regular field visits for water sampling time for water quality study, the identification of biodiversity species were documented.

\section{Results \& discussion:-}

In the Peri urban area of Bengaluru, water bodies covers have attained an important ecological status as the lakes have turned into lentic-closed water habitats. These lakes form a characteristic extremely rare system and habituated ddifferent types of biodiversity.

The following are the biodiversity species were identified in the studied lakes. Herbs and Shrubs: A maximum of around 37 species of Herbs and shrubs were recorded in Byramangala lake and a minimum of 28 species in Bandematta lake (Annexure 2). Aquatic Plants: A maximum of around 13 species of aquatic plants were recorded in both Hosakerehalli lake and Dorekere lake and a minimum of 3 species in Bandematta lake (Annexure 3). Trees: A maximum of around 21 species of trees were recorded in Hosakerehalli lake and a minimum of 11 species in Margondanahalli Hosakere lake (Annexure 1). Reptiles: Around 6 species of Reptiles were recorded in Bandematta lake, Hosakerehalli lake and Dorekere lake and only 3 species were recorded in Bandematta lake (Annexure 4). Odonates: 16 species of Odonates were recorded in Kommaghatta lake and 7 species in Subramanayapura lake (Annexure 5). Birds: A maximum of around 38 species of Birds were recorded in Kommaghatta lake and a minimum of 19 species in Hosakerehalli lake (Annexure 6). Butterflies: 24 species of Butterflies were recorded in Dorekere lake and 10 species in Byramangala lake (Annexure 7). Mammals: Around 9 species of Mammals were recorded in Margondanahalli Hosakere lake and only 4 species in Hosakerehalli lake (Annexure 9). Insects: 21 species of Insects were recorded in Bandematta lake and 12 species in Kommaghatta lake (Annexure 10). Fishes: 10 species of Fishes were recorded in Kommaghatta lake and only one species in Bandematta lake (Annexure 8).

The following plate details are the main examples of Flora and Fauna identified with common names.

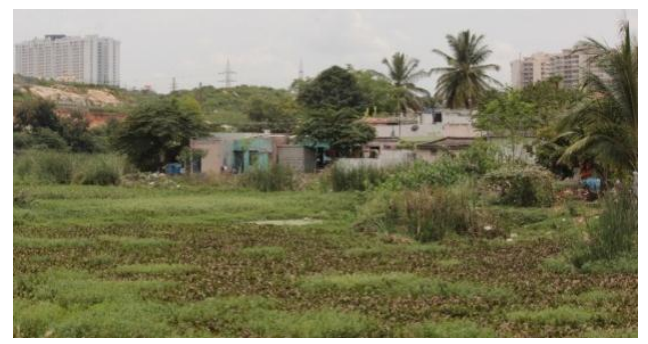

Plate 1: Common Cattail and Alligator weed

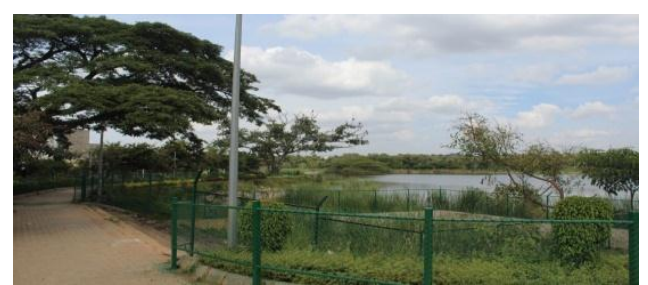

Plate 3: Rain tree

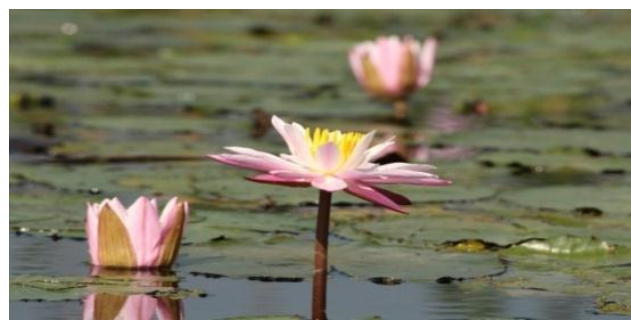

Plate 2: Indian lotus

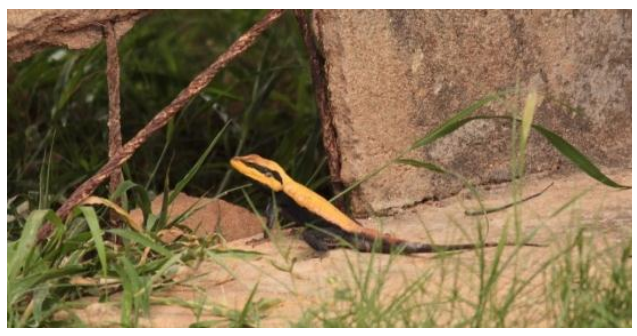

Plate 4: Peninsular Rock Agama 


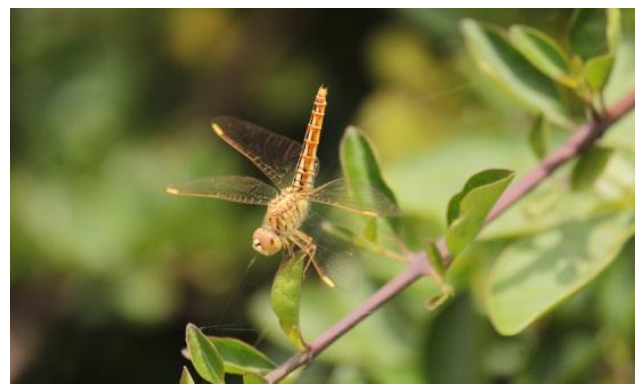

Plate 5: Ditch Jewel

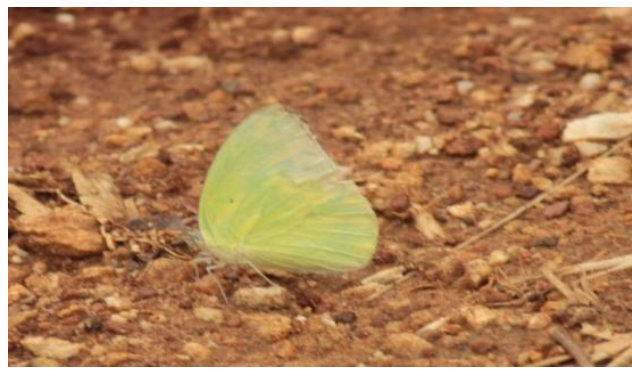

Plate 7: Common Emigrant

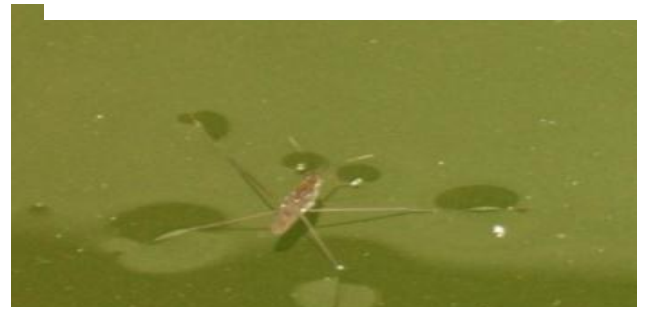

Plate 9: Water Strider

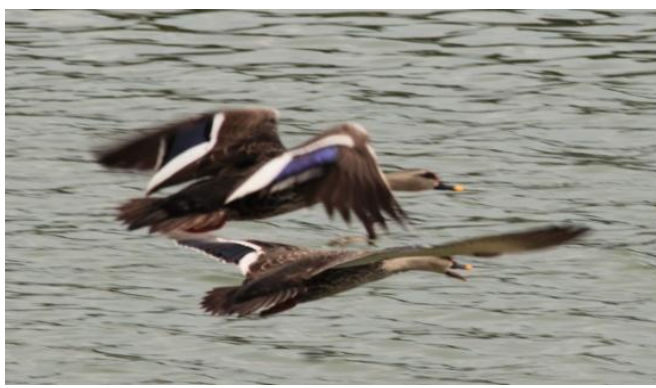

Plate 6: Spot Billed duck

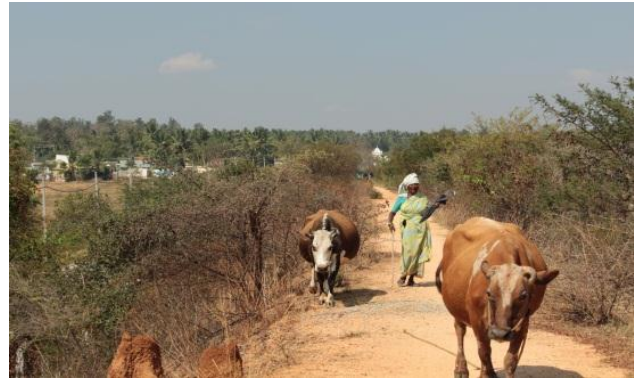

Plate 8: Cattles

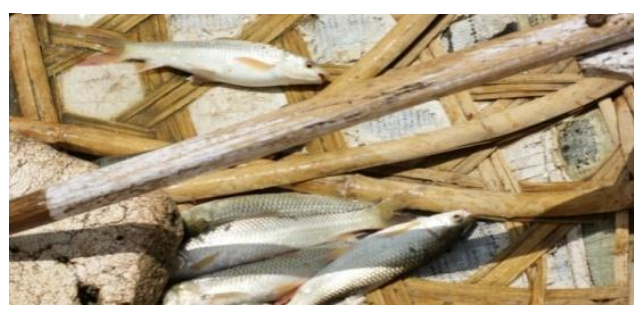

Plate 10: Mrigal

The overall recorded species of Flora and Fauna in the decreasing order are as follows: Kommaghatta lake (171 species)> Dorekere lake (169 species)> Margondanahalli Hosakere lake (161 species)> Subramanayapura lake (159 species) > Byramangala lake (157 species) > Bandematta lake (148 species)> Hosakerehalli lake (146 species).It was observed that, the various decaying weeds in the lakes formed floating vegetation islands/mats which deteriorate habitat quality and also interfere with foraging activities of water birds as observed by Bhatnagar et al., (2007). Further birds' nests were not found in these weeds. Mukherjee et al., (2002) reported that aquatic weeds and short vegetation are not suitable for nesting. In addition to the weeds, other factors affecting the water bird populations in various lakes were due to fishing.

Kommaghatta Lake and Dorekere Lakes were found to be highest in species diversity; this is due to recent rejuvenation and plantation programmes carried out in these lakes. These two lakes are having the Islands and were recorded highest Bird species diversity. The local associations and citizens have also adopted the plants for the maintenance and protection. Next species diversity was recorded in Margondanahalli Hosakere as it is in the less populated zone; the lake is situated away from the village and near to Sulikere reserve forest. The shorelines of these lakes are also well maintained and hence, the recorded species were found maximum in these lakes. The lakes are situated in urbanised and industrialised area and the shorelines of these lakes are facing issues like encroachment, sewage entry and waste dumping. Similarly the study of Erickson (2011) revealed that, biodiversity of lakes helped in cleaning of larger water bodies by removing the nitrate pollutants. Research study of Meli et al.,(2014) revealed that, ecological restoration of wetlands will increases both biodiversity and provides many ecosystem services such as water quality. 


\section{Bio diversity in and around lakes and its management}

The lakes in the study area are maintained by the authorities such as BDA, BBMP and Zilla Panchayath of Bengaluru Urban district. The authorities should maintain buffer zones around the lakes and at the upstream of lakes, which should be made mandatory for the conservation of biodiversity.

In all the lakes in the study area only horticultural Plantations have been planted, native species are not maintained. The existing conditions of lakes including water quality can be improved by protecting the native species in and around lakes. The regular monitoring and surveys using GIS and Remote sensing techniques will help in giving vegetation cover database of lakes.

Sustainable management of water and sanitation for all lakes ensure fresh water, help replenish ground aquifers, and purify and filter harmful waste from water such as fertilizers and pesticides, as well as heavy metals and toxins. There is a need to conserve and maintain the vegetation of the wetlands that help in absorbing these harmful toxins. Sustainable management of lakes can also be achieved through participation of local communities, nature conservation organisations and fishing associations as cooperation partners in the lake management. Policy incentives to encourage nature conservation are emerging around the world, and yet this trend remains handicapped by a lack of understanding of the economic benefits of conserving Biodiversity, therefore it is recommended that appropriate policy incentivise are to be developed and employed.

\section{Conculusion:-}

The Bengaluru periphery area experiencing accelerated growth with changes in ecosystems, land use and governance leading to impacts on freshwater ecosystems and its biodiversity. Managing of these area lakes is a challenging mission for the sustainable development of Bengaluru. Biodiversity is to be considered as a prime point of conservation by adopting the good lake shoreline management techniques or practices by the dogmatic authorities such as maintaining buffer zones around the lakes and at the upstream of lakes, the native species in and around lakes, regular monitoring and surveys using GIS and Remote sensing techniques, by adopting Sustainable management of water and sanitation encouraging the Policy incentives for conserving the lakes.

Annexure 1:-List of Tree species recorded during the study period in and around lakes

\begin{tabular}{|c|c|c|c|c|c|c|c|c|}
\hline name & $\begin{array}{l}\text { C } \\
\text { ommon } \\
\text { Name }\end{array}$ & $\begin{array}{r}\text { Ma } \\
\text { rgondanahal } \\
\text { li Hosakere }\end{array}$ & $\begin{array}{l}\text { K } \\
\text { ommagha } \\
\text { tta }\end{array}$ & $\begin{array}{l}\text { andemat } \\
\text { ta }\end{array}$ & $\begin{array}{l}\text { osaker } \\
\text { e- } \\
\text { alli }\end{array}$ & $\begin{array}{l}\text { oreker } \\
\mathrm{e}\end{array}$ & $\begin{array}{l}\text { ubraman } \\
\text { a- } \\
\text { apura }\end{array}$ & $\begin{array}{l}\text { Bramang } \\
\text { ala }\end{array}$ \\
\hline $\begin{array}{ll}\text { giosa } & \text { Ficusreli } \\
\end{array}$ & epal Tree $^{\mathrm{Pe}}$ & + & + & 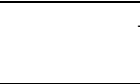 & & & + & + \\
\hline $\begin{array}{ll} & \text { Bamboo } \\
\text { sp. } & \end{array}$ & amboo & + & + & 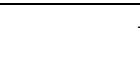 & & & + & + \\
\hline $\begin{array}{l}\text { Phoenix } \\
\text { sylvestris }\end{array}$ & $\begin{array}{r}\mathrm{Ja} \\
\mathrm{ck} \text { fruit tree }\end{array}$ & + & + & . & & & + & + \\
\hline catechu & $\begin{array}{r}\mathrm{Ar} \\
\text { ecanut tree }\end{array}$ & - & - & 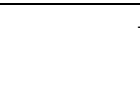 & & & & + \\
\hline $\begin{array}{l}\text { Pongami } \\
\text { apinnata }\end{array}$ & $\begin{array}{ll} & \text { Po } \\
\text { ngam } & \\
\end{array}$ & + & + & . & & & + & + \\
\hline ifera & $\begin{array}{ll} & \mathrm{C} \\
\text { oconut } \\
\text { Tree }\end{array}$ & + & + & 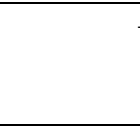 & & & + & + \\
\hline$u_{\text {usindica }}^{\text {Tamarind }}$ & $\begin{array}{l}\text { Ta } \\
\text { marind } \\
\text { Tree }\end{array}$ & + & + & . & & & + & + \\
\hline $\begin{array}{ll} & \text { Samanea } \\
\text { saman } & \end{array}$ & ain Tree $^{\mathrm{R}}$ & - & - & . & & & - & + \\
\hline $\begin{array}{l}\text { Azadirac } \\
\text { htaindica }\end{array}$ & eem Tree & + & - & 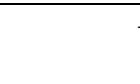 & & & + & + \\
\hline Tectonag & $\mathrm{Te}$ & + & + & . & & & + & - \\
\hline
\end{tabular}




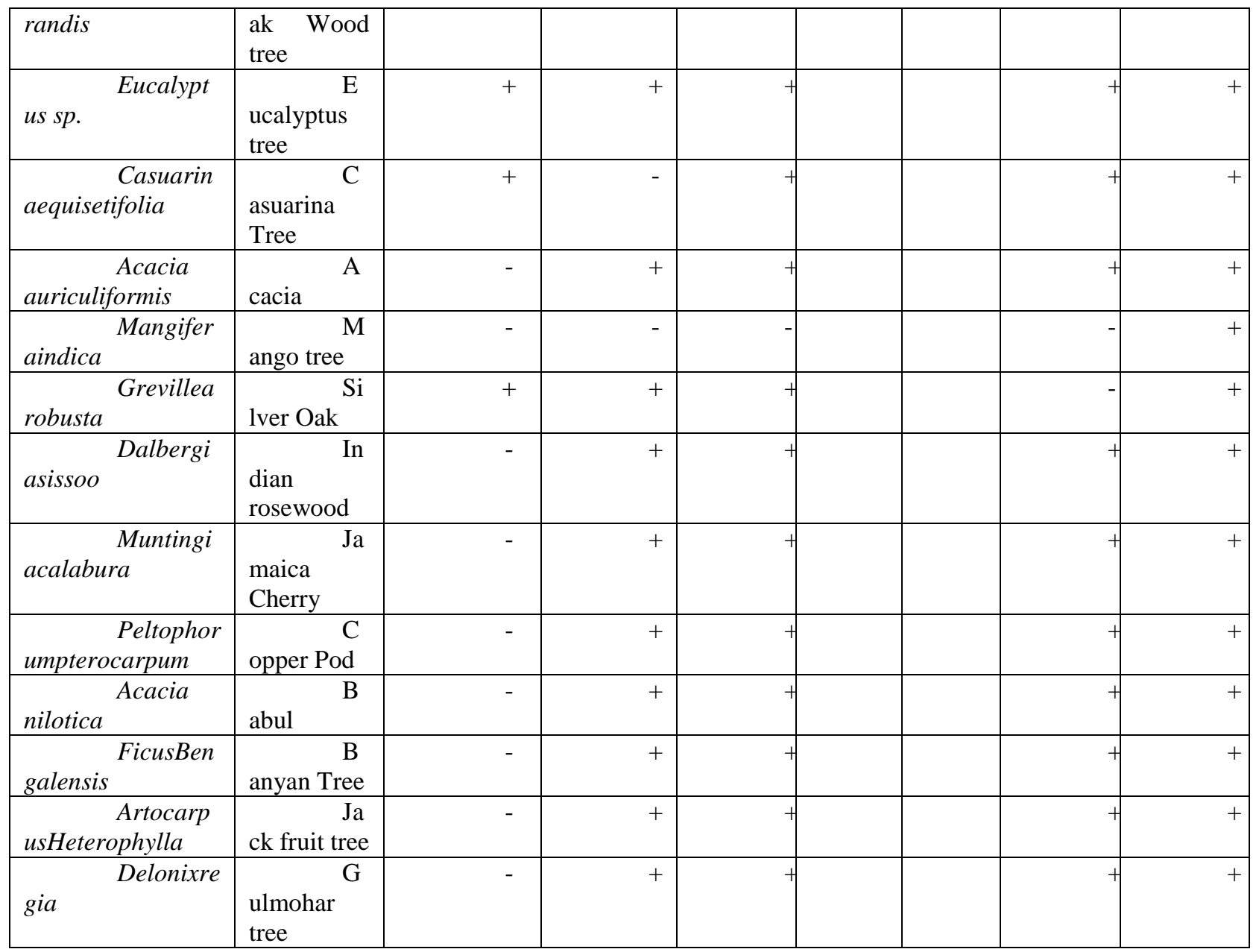

Annexure 2:-List of Herbs and Shrubs species recorded during the study period in and around lakes

\begin{tabular}{|c|c|c|c|c|c|c|c|c|}
\hline name & $\begin{array}{l}\text { mo } \\
\text { Namen }\end{array}$ & $\begin{array}{r}\text { Mar } \\
\text { gondanahall } \\
\text { i Hosakere }\end{array}$ & $\underset{\text { ta }}{\text { ommaghat }}$ & $\begin{array}{c}\text { and } \\
\text { e } \\
\text { atta }\end{array}$ & $\begin{array}{c}\text { osaker } \\
\quad \text { e } \\
\text { alli }\end{array}$ & $\begin{array}{l}\text { ore } \\
\text { ere }\end{array}$ & $\begin{array}{cr} & S \\
\text { ubramana } & \\
\text { ya } & \text { p } \\
\text { ura } & \end{array}$ & $\begin{array}{c}\text { Bramang } \\
\text { ala }\end{array}$ \\
\hline $\begin{array}{l}\text { Cynodond } \\
\text { actylon }\end{array}$ & ass $\quad \mathrm{Gr}$ & + & + & & & & + & + \\
\hline $\begin{array}{ll}\text { Mudica } & \text { Mimosa } \\
\text { pula }\end{array}$ & $\begin{array}{r}\text { To } \\
\text { uch Me not }\end{array}$ & + & + & & & & + & + \\
\hline $\begin{array}{l}\text { Borrerias } \\
\text { tricta }\end{array}$ & $\begin{array}{ll} & \mathrm{Ta} \\
\text { rtaval } & \end{array}$ & + & + & & & & + & + \\
\hline $\begin{array}{l}\text { Partheniu } \\
\text { mhysterophorus }\end{array}$ & thenium & - & + & & & & + & - \\
\hline a hirta Euphorbi & $\begin{array}{l}\text { Ca } \\
\text { ts hair, } \\
\text { asthma } \\
\text { weed }\end{array}$ & + & + & & & & - & - \\
\hline carnea & $\begin{array}{l}\text { Pin } \\
\mathrm{k} \text { morning } \\
\text { glory }\end{array}$ & + & + & & & & - & + \\
\hline $\begin{array}{l}\text { Amaranth } \\
\text { usspinosus }\end{array}$ & ny $\quad$ Spi & + & + & & & & + & + \\
\hline
\end{tabular}




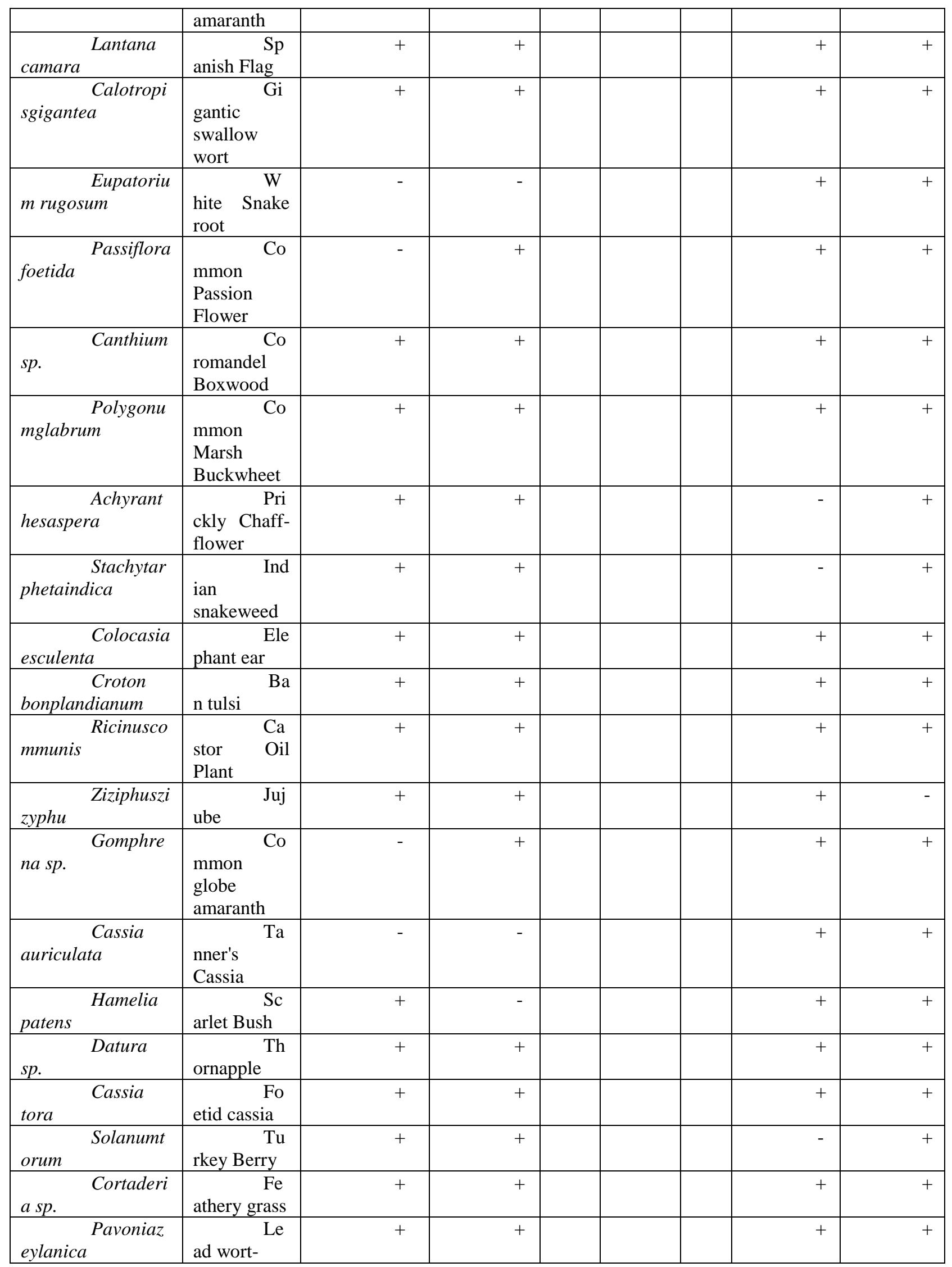




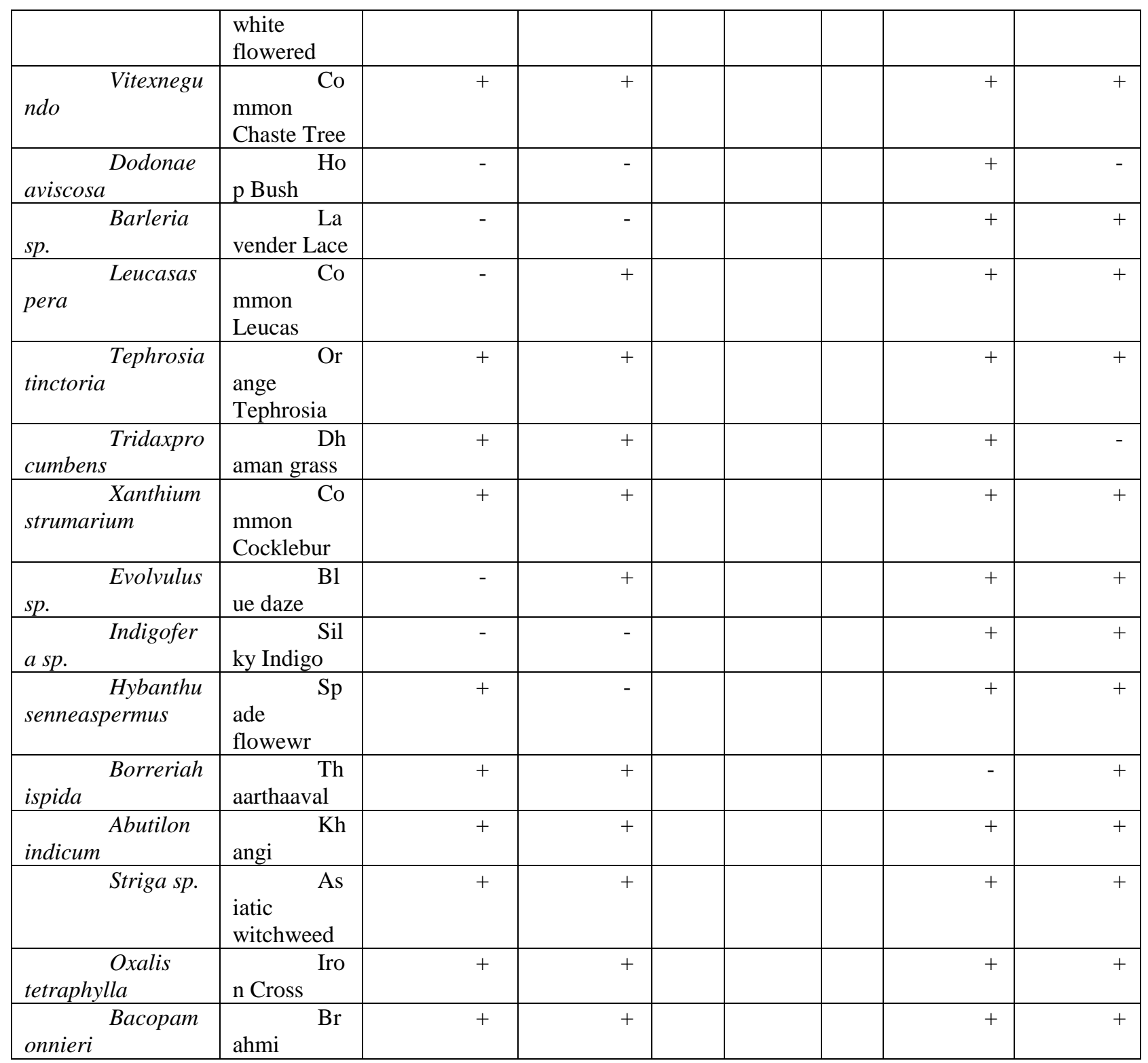

Annexure 3:-List of Aquatic species recorded during the study period in studied lakes

\begin{tabular}{|c|c|c|c|c|c|c|c|c|}
\hline $\begin{array}{l}\text { Scientifi } \\
\text { c name }\end{array}$ & $\begin{array}{c}\mathrm{C} \\
\text { ommon } \\
\text { Name }\end{array}$ & $\begin{array}{c}\text { Ma } \\
\text { rgondanah } \\
\text { alli } \\
\text { Hosakere }\end{array}$ & $\begin{array}{c}\text { ommagh } \\
\text { atta }\end{array}$ & $\begin{array}{c}\text { andema } \\
\text { tta }\end{array}$ & $\begin{array}{c}\text { osaker } \\
\text { e- } \\
\text { alli }\end{array}$ & $\begin{array}{c}\text { oreke } \\
\text { re }\end{array}$ & $\begin{array}{c}\text { ubrama } \\
\text { na- } \\
\text { apura }\end{array}$ & $\begin{array}{c}\text { yramang } \\
\text { ala }\end{array}$ \\
\hline Typha sp. & $\begin{array}{l}\mathrm{C} \\
\text { ommon } \\
\text { Cattail }\end{array}$ & - & + & - & & & + & + \\
\hline $\begin{array}{l}\text { Hydrillav } \\
\text { erticillata }\end{array}$ & $\begin{array}{ll}\text { ydrilla } & \mathrm{H} \\
\end{array}$ & + & + & & & & + & - \\
\hline minor & $\begin{array}{l}\quad \text { L } \\
\text { esser } \\
\text { Duckweed }\end{array}$ & + & + & & & & + & - \\
\hline $\begin{array}{ll} & \text { Nymphae } \\
\text { a sp. } & \end{array}$ & $\begin{array}{rr} & R \\
\text { ed } \quad \text { water }\end{array}$ & + & - & & & & + & - \\
\hline
\end{tabular}




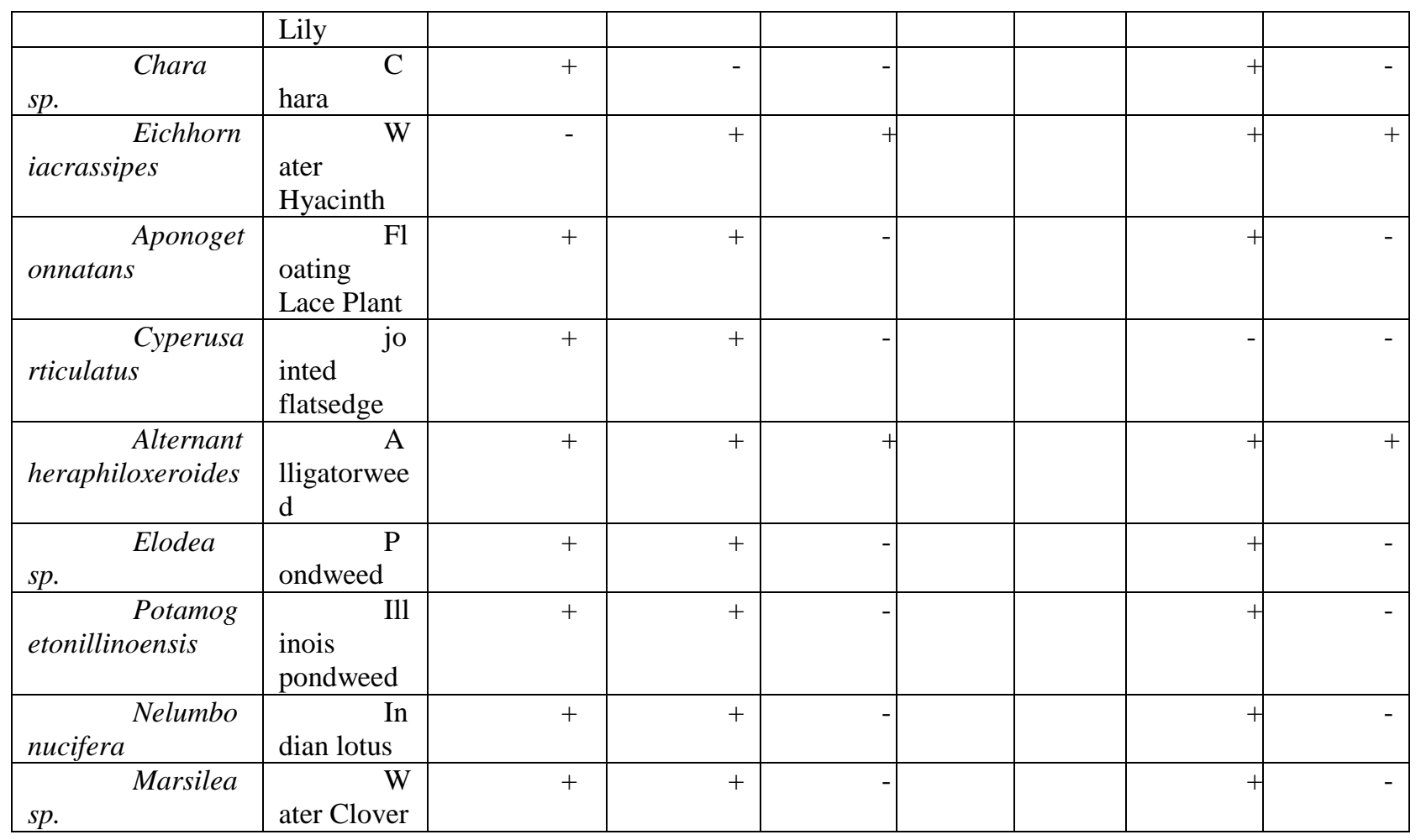

Annexure 4:-List of Reptiles species recorded during the study period in studied lakes

\begin{tabular}{|c|c|c|c|c|c|c|c|c|}
\hline c name & $\begin{array}{l}\text { Cmmon } \\
\text { Name }\end{array}$ & $\begin{array}{r}\text { Ma } \\
\text { rgondanahal } \\
\text { li Hosakere }\end{array}$ & $\begin{array}{c}\mathrm{K} \\
\text { tta }\end{array}$ & $\begin{array}{c}\text { andemat } \\
\text { ta }\end{array}$ & $\begin{array}{c}\text { osaker } \\
\mathrm{e} \\
\text { alli } \\
\end{array}$ & $\begin{array}{c}\text { oreker } \\
\mathrm{e}\end{array}$ & $\begin{array}{cc} & S \\
\text { ybramana } & \\
& p \\
\text { ura } & \\
\end{array}$ & $\begin{array}{c}\text { yramang } \\
\text { ala }\end{array}$ \\
\hline $\begin{array}{l}\text { Psammo } \\
\text { philusdorsalis }\end{array}$ & $\begin{array}{l}\text { Pe } \\
\text { ninsular } \\
\text { Rock } \\
\text { Agama } \\
\end{array}$ & + & + & + & + & + & + & + \\
\hline atra & $\begin{array}{l}\text { So } \\
\text { thern Rock } \\
\text { Agama }\end{array}$ & + & + & + & + & + & + & + \\
\hline $\begin{array}{l}\text { Calotesv } \\
\text { ersicolor }\end{array}$ & $\begin{array}{l}\text { C } \\
\text { ommon } \\
\text { Garden } \\
\text { Lizard } \\
\end{array}$ & + & + & + & + & + & + & + \\
\hline carinata & $\begin{array}{l}\text { C } \\
\text { ommon } \\
\text { Skink } \\
\end{array}$ & - & - & + & + & + & - & + \\
\hline Najanaja & $\begin{array}{r}\text { In } \\
\text { dian Cobra }\end{array}$ & + & - & - & - & - & - & - \\
\hline $\begin{array}{l}\text { Hoploba } \\
\text { trachustigerinus }\end{array}$ & $\begin{array}{lc} & \text { In } \\
\text { dian } & \text { Bull } \\
\text { Frog } & \\
\end{array}$ & + & + & + & + & + & + & + \\
\hline $\begin{array}{l}\text { Bufomel } \\
\text { anostictus }\end{array}$ & $\begin{array}{l}\text { C } \\
\text { ommon } \\
\text { Indian } \\
\text { Toad }\end{array}$ & - & - & + & + & + & - & + \\
\hline
\end{tabular}

Note: PRESENT (+); ABSENT (-) 
Annexure 5:-List of Odonates species recorded during the study period in studied lakes

\begin{tabular}{|c|c|c|c|c|c|c|c|c|}
\hline name & $\begin{array}{ll} & \text { Co } \\
\text { mamon } & \\
\text { Name }\end{array}$ & $\begin{array}{r}\text { Ma } \\
\text { rgondanahal } \\
\text { li Hosakere }\end{array}$ & $\begin{array}{c}\mathrm{K} \\
\text { ommaghat } \\
\text { ta }\end{array}$ & $\begin{array}{l}\text { ande } \\
\text { atta }\end{array}$ & $\begin{array}{c}\text { osaker } \\
\text { e } \\
\text { alli }\end{array}$ & $\begin{array}{l}\text { ore } \\
\text { ere }\end{array}$ & 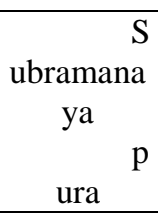 & $\begin{array}{r}\text { B } \\
\text { yramanga } \\
\text { la }\end{array}$ \\
\hline $\begin{array}{ll} & \text { Trithemis } \\
\text { aurora } & \end{array}$ & $\begin{array}{ll} & \mathrm{Cr} \\
\text { imson } & \\
\text { Marsh } & \\
\text { Glider } & \\
\end{array}$ & + & + & & & & + & + \\
\hline $\begin{array}{l}\text { Brachythemis } \\
\text { contaminata }\end{array}$ & $\begin{array}{l}\text { Di } \\
\text { tch Jewel }\end{array}$ & + & + & & & & + & + \\
\hline $\begin{array}{l}\text { Brachydiplax } \\
\text { sobrina }\end{array}$ & $\begin{array}{lr} & \text { Lit } \\
\text { tle } & \text { Blue } \\
\text { Marsh } & \\
\text { Hawk } & \\
\end{array}$ & + & + & & & & + & + \\
\hline $\begin{array}{l}\text { Ictinogomph } \\
\text { usrapax. }\end{array}$ & $\begin{array}{l}\text { Co } \\
\text { mmon } \\
\text { Clubtail }\end{array}$ & - & + & & & & - & + \\
\hline $\begin{array}{l}\text { Rhyothemisv } \\
\text { ariegata }\end{array}$ & $\begin{array}{l}\text { Co } \\
\text { mmon } \\
\text { picture } \\
\text { wing } \\
\end{array}$ & - & - & & & & - & - \\
\hline bina Orthetrumsa & $\begin{array}{l}\text { Gr } \\
\text { een Marsh } \\
\text { Hawk }\end{array}$ & + & + & & & & - & - \\
\hline $\begin{array}{ll} & \text { Diplacodestri } \\
\text { vialis } & \end{array}$ & $\begin{array}{l}\text { Gr } \\
\text { ound } \\
\text { Skimmer }\end{array}$ & + & - & & & & - & + \\
\hline $\begin{array}{ll}\text { ervilia } & \text { Crocothemiss }\end{array}$ & $\begin{array}{l}\text { Ru } \\
\text { ddy Marsh } \\
\text { Skimmer }\end{array}$ & + & - & & & & - & - \\
\hline $\begin{array}{l}\text { Rhodothemis } \\
\text { rufa }\end{array}$ & $\begin{array}{l}\text { Ru } \\
\text { fous Marsh } \\
\text { Glider }\end{array}$ & - & + & & & & - & - \\
\hline $\begin{array}{l}\text { Orthetrumluz } \\
\text { onicum }\end{array}$ & $\begin{array}{l}\text { Tri } \\
\text { coloured } \\
\text { Marsh } \\
\text { Hawk }\end{array}$ & - & + & & & & + & - \\
\hline ta Tramealimba & $\begin{array}{l}\text { Bl } \\
\text { ack marsh } \\
\text { trotter }\end{array}$ & - & + & & & & - & + \\
\hline Anaxguttatus & $\begin{array}{l} \\
\text { ue } \quad \text { Bl } \\
\text { green } \\
\text { dailed } \\
\text { darner }\end{array}$ & - & + & & & & - & + \\
\hline $\begin{array}{l}\text { Paragomphu } \\
\text { slineatus }\end{array}$ & $\begin{array}{l}\text { Co } \\
\text { mmon } \\
\text { Hooktail }\end{array}$ & + & + & & & & - & - \\
\hline $\begin{array}{l}\text { Trithemispall } \\
\text { idinervis }\end{array}$ & $\begin{array}{l}\text { Lo } \\
\text { ng legged } \\
\text { marsh } \\
\text { glider }\end{array}$ & + & + & & & & - & - \\
\hline Pseudagrion & $\mathrm{B} 1$ & + & - & & & & - & - \\
\hline
\end{tabular}




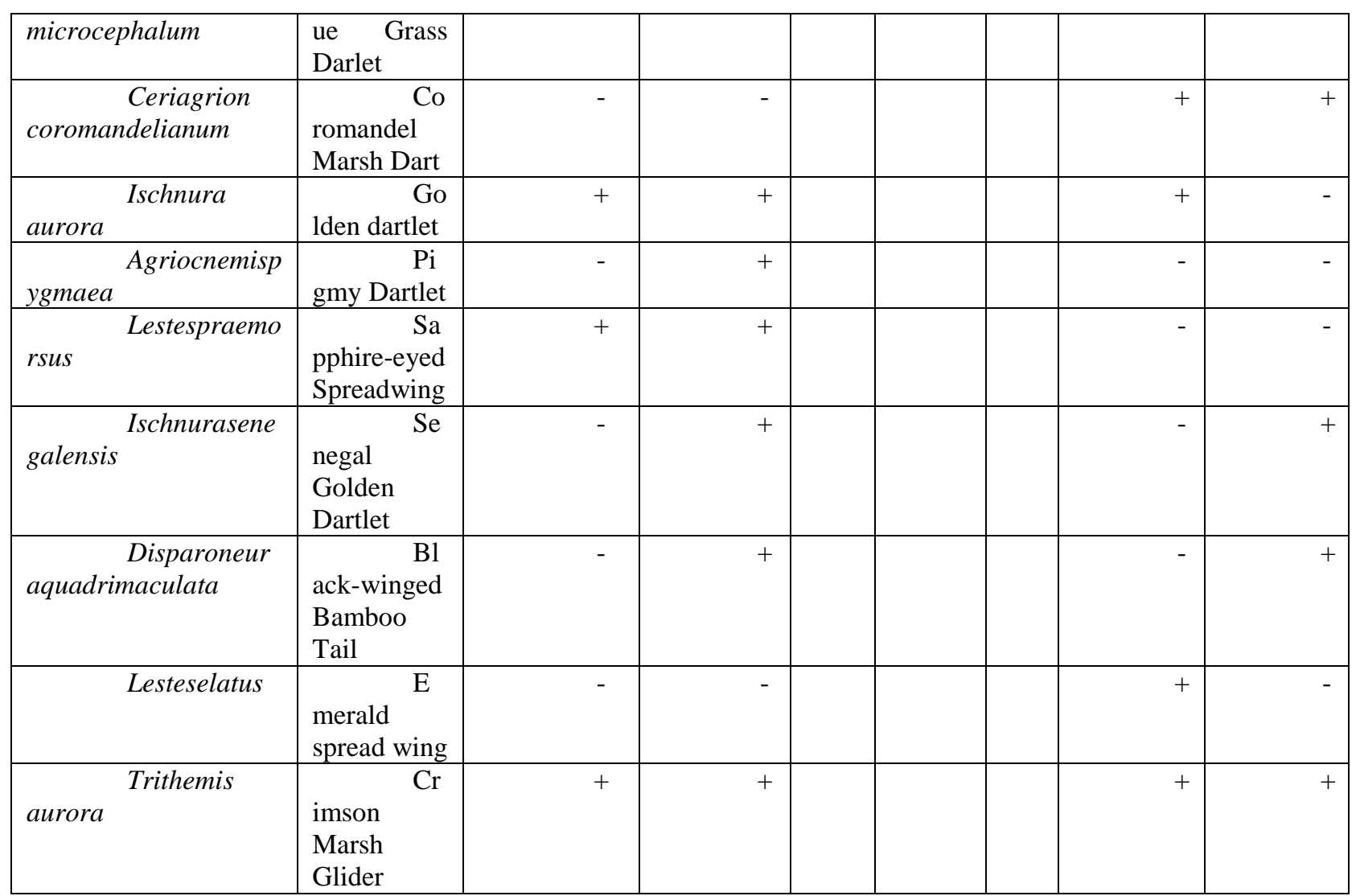

Note: PRESENT (+); ABSENT (-)

Annexure 6:-List of Birds species recorded during the study period in studied lakes

\begin{tabular}{|c|c|c|c|c|c|c|c|c|}
\hline c Name ${ }^{\text {Scientifi }}$ & Name Common & $\begin{array}{c}\text { Margonda } \\
\text { nahalli } \\
\text { Hosakere }\end{array}$ & $\begin{array}{c}\text { Kommag } \\
\text { hatta }\end{array}$ & $\begin{array}{l}\text { Bande } \\
\text { matta }\end{array}$ & $\begin{array}{c}\text { Hosak } \\
\text { ere- } \\
\text { halli } \\
\end{array}$ & $\begin{array}{l}\text { Dore } \\
\text { kere }\end{array}$ & $\begin{array}{l}\text { Subram } \\
\text { ana- } \\
\text { yapura }\end{array}$ & $\begin{array}{c}\text { Byrama } \\
\text { ngala }\end{array}$ \\
\hline $\begin{array}{l}\text { Anaspoe } \\
\text { cilorhyncha }\end{array}$ & $\begin{array}{r}\text { Spot } \\
\text { Billed Duck }\end{array}$ & + & + & & & & & - \\
\hline sindicus & $\begin{array}{c}\text { Red } \\
\text { wattled lapwing }\end{array}$ & - & + & & & & & - \\
\hline $\begin{array}{l}\text { Hydrop } \\
\text { hasianuschirurgu } \\
\text { s }\end{array}$ & $\begin{array}{l}\text { Pheasant } \\
\text { tailed Jacana }\end{array}$ & - & + & & & & & + \\
\hline $\begin{array}{r}\text { Himant } \\
\text { opushimantopus }\end{array}$ & $\begin{array}{r}\text { Black } \\
\text { Winged Stilt } \\
\end{array}$ & - & + & & & & & + \\
\hline garzetta & $\begin{array}{ll}\text { Egret } & \text { Little } \\
\end{array}$ & + & + & & & & & - \\
\hline $\begin{array}{l}\text { streptop } \\
\text { eliachinensis }\end{array}$ & $\begin{array}{ll}\text { Spotted } \\
\text { dove }\end{array}$ & - & + & & & & & - \\
\hline $\begin{array}{ll} & \text { Alcedoa } \\
\text { tthis } & \\
\end{array}$ & $\begin{array}{l}\text { Common } \\
\text { Kingfisher } \\
\end{array}$ & + & + & & & & & + \\
\hline udis & $\begin{array}{l}\text { Pied } \\
\text { kingfisher }\end{array}$ & + & + & & & & & + \\
\hline $\begin{array}{l}\text { Halcyon } \\
\text { smyrnensis }\end{array}$ & $\begin{array}{l}\text { White } \\
\text { throated king } \\
\text { fisher }\end{array}$ & + & + & & & & & + \\
\hline $\begin{array}{l}\text { Merops } \\
\text { orientalis } \\
\end{array}$ & $\begin{array}{l}\text { Green } \\
\text { Bee eater }\end{array}$ & + & + & & & & & - \\
\hline
\end{tabular}




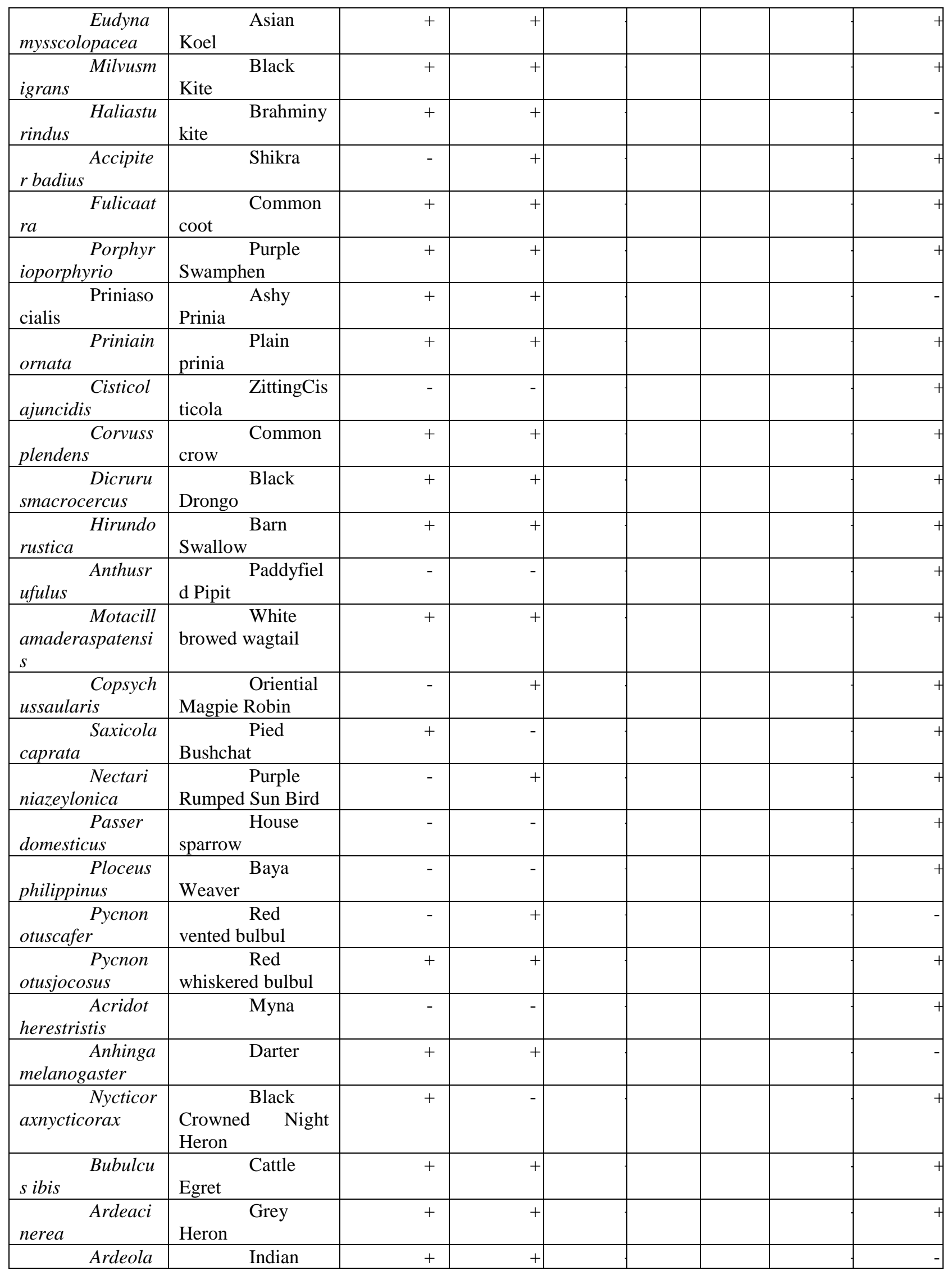




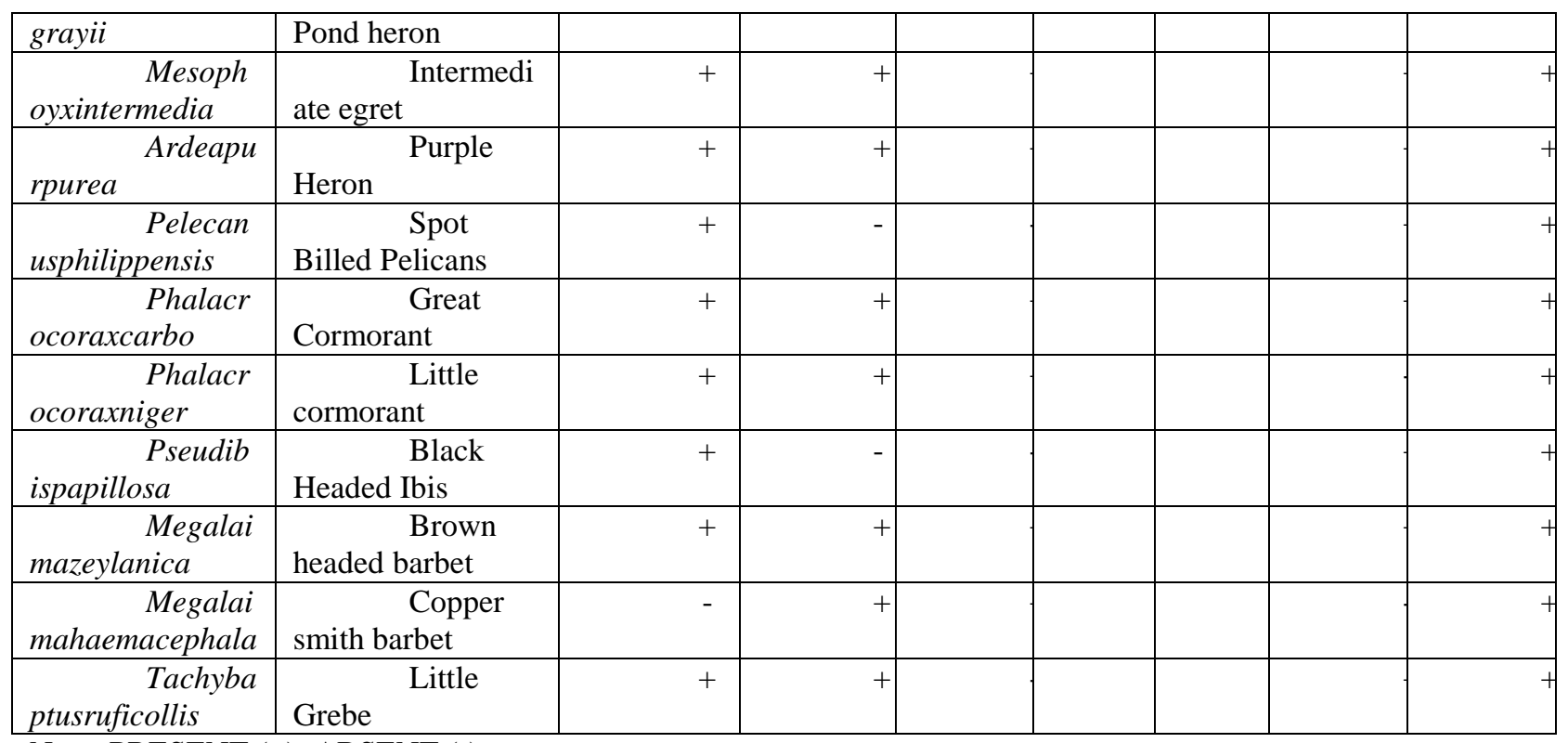

Note: PRESENT (+); ABSENT (-)

Annexure 7:-List of Butterflies species recorded during the study period in studied lakes

\begin{tabular}{|c|c|c|c|c|c|c|c|c|}
\hline ific Name & $\begin{array}{l}\text { ommo } \\
\text { n } \\
\text { Name }\end{array}$ & $\begin{array}{c}\text { M } \\
\text { argondana } \\
\text { halli } \\
\text { Hosakere }\end{array}$ & $\begin{array}{c}\text { ommagh } \\
\text { atta }\end{array}$ & $\underset{\text { tta }}{\text { andema }}$ & $\begin{array}{c}\text { H } \\
\text { osakereh } \\
\text { alli }\end{array}$ & $\begin{array}{l}\text { oreke } \\
\text { re }\end{array}$ & $\begin{array}{r}\text { Sub } \\
\text { ramanayap } \\
\text { ura }\end{array}$ & $\begin{array}{c}\text { yramang } \\
\text { ala }\end{array}$ \\
\hline $\begin{array}{l}\text { Castal } \\
\text { iusrosimon }\end{array}$ & $\begin{array}{l}\text { ommon } \\
\text { Pierrot }\end{array}$ & + & + & $t$ & + & & + & - \\
\hline $\begin{array}{l}\text { Freyer } \\
\text { iatrochylus }\end{array}$ & $\begin{array}{l}\text { rassjew } \\
\text { el }\end{array}$ & + & + & + & + & & + & - \\
\hline${ }^{\text {Jamid }}$ & $\begin{array}{l}\text { ommon } \\
\text { Cerule } \\
\text { an }\end{array}$ & + & + & + & + & & - & - \\
\hline $\begin{array}{r}\text { Lampi } \\
\text { desboeticus }\end{array}$ & ea Blue & - & + & + & - & & - & - \\
\hline esplinius $^{\text {Leptot }}$ & $\begin{array}{l}\text { ebra } \\
\text { Blue }\end{array}$ & + & + & + & - & & + & + \\
\hline $\begin{array}{r}\text { Pseud } \\
\text { ozizeeriamaha }\end{array}$ & $\begin{array}{l}\text { ale } \\
\text { Grass } \\
\text { Blue }\end{array}$ & - & + & - & - & & + & - \\
\hline $\begin{array}{l}\text { Ariadn } \\
\text { e merione }\end{array}$ & $\begin{array}{l}\text { ommon } \\
\text { castor }\end{array}$ & - & + & + & + & & + & - \\
\hline $\begin{array}{l}\text { Danau } \\
\text { schrysippus }\end{array}$ & $\begin{array}{l}\text { lainTig } \\
\text { er }\end{array}$ & + & + & - & - & & - & + \\
\hline $\begin{array}{r}\text { Elymni } \\
\text { ashypermenstra }\end{array}$ & $\begin{array}{l}\text { ommon } \\
\text { Palmfl } \\
\text { y }\end{array}$ & - & - & + & - & & + & + \\
\hline
\end{tabular}




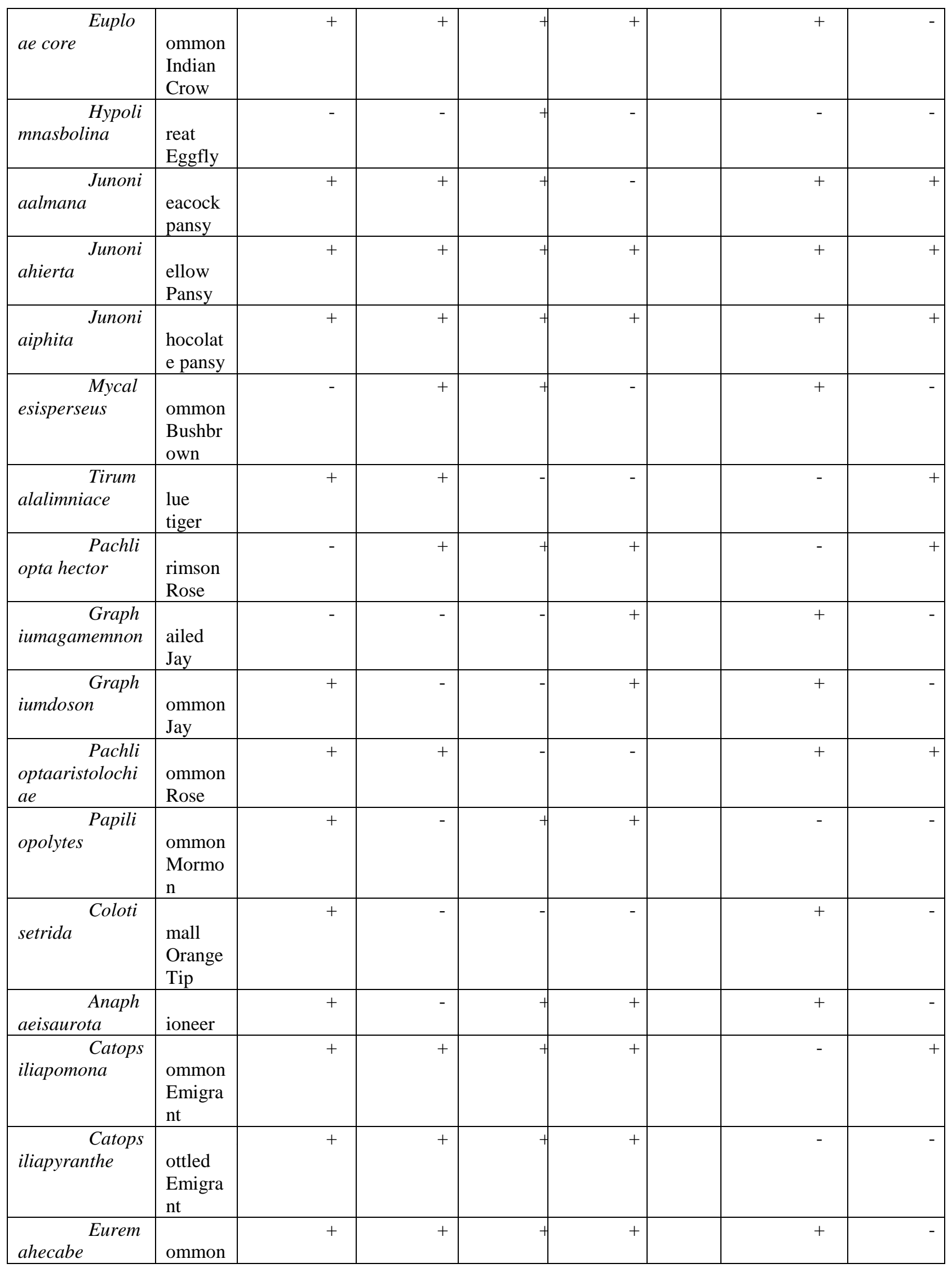




\begin{tabular}{|c|l|l|r|r|r|r|r|r|}
\hline & $\begin{array}{l}\text { Grass } \\
\text { Yellow }\end{array}$ & & & & & & & \\
\hline ianina Leptos & syche & + & + & - & + & & & - \\
\hline
\end{tabular}

Note: PRESENT (+); ABSENT (-) 
Annexure 8:-List of Fish species recorded during the study period in studied lakes

\begin{tabular}{|c|c|c|c|c|c|c|c|c|}
\hline Name & $\begin{array}{l}\text { ommon } \\
\text { Name }\end{array}$ & $\begin{array}{c}\text { Margonda } \\
\text { nahalli } \\
\text { Hosakere }\end{array}$ & $\begin{array}{c}\text { Kommag } \\
\text { hatta }\end{array}$ & $\begin{array}{l}\text { Bande } \\
\text { matta }\end{array}$ & $\begin{array}{c}\text { Hosak } \\
\text { ere- } \\
\text { halli }\end{array}$ & $\begin{array}{l}\text { Dore } \\
\text { kere }\end{array}$ & $\begin{array}{c}\text { Subram } \\
\text { ana- } \\
\text { yapura }\end{array}$ & $\begin{array}{c}\text { Byrama } \\
\text { ngala }\end{array}$ \\
\hline Catlacatl & dian carp & + & + & - & + & - & + & + \\
\hline Labeoroh & $\begin{array}{r}\mathrm{R} \\
\text { ohiorrohu }\end{array}$ & + & + & - & - & + & - & - \\
\hline mrigala & $\begin{array}{l}\text { M } \\
\text { rigal }\end{array}$ & + & + & - & - & + & - & + \\
\hline $\begin{array}{l}\text { Cyprinusc } \\
\text { arpio }\end{array}$ & $\begin{array}{l}\text { ommon } \\
\text { carp }\end{array}$ & + & + & - & - & + & + & - \\
\hline $\begin{array}{r}\text { Hypophth } \\
\text { almichthysmolitrix }\end{array}$ & lver carp & + & + & - & - & + & - & - \\
\hline $\begin{array}{l}\text { Amblypha } \\
\text { ryngodonmola }\end{array}$ & $\begin{array}{r}\mathrm{M} \\
\text { olacarplet }\end{array}$ & + & + & - & + & + & - & - \\
\hline $\begin{array}{l}\text { Etropluss } \\
\text { uratensis }\end{array}$ & earl Spot & + & + & - & - & - & - & - \\
\hline $\begin{array}{l}\text { Channam } \\
\text { arulius }\end{array}$ & $\begin{array}{ll} & \mathrm{M} \\
\text { urrels } & \end{array}$ & + & + & - & - & + & - & + \\
\hline $\begin{array}{r}\text { Oreochro } \\
\text { mismossambicus }\end{array}$ & lapia $\quad \mathrm{Ti}$ & + & + & + & + & + & + & + \\
\hline $\begin{array}{l}\text { Heteropn } \\
\text { eustesfossilis }\end{array}$ & $\begin{array}{l}\text { C } \\
\text { atfishes }\end{array}$ & - & + & - & + & - & - & + \\
\hline $\begin{array}{l}\text { Channap } \\
\text { unctatus }\end{array}$ & $\begin{array}{l}\text { potted } \\
\text { snake } \\
\text { head } \\
\text { (Korava) }\end{array}$ & + & - & - & - & + & + & + \\
\hline riepinus & $\begin{array}{l}\text { A } \\
\text { frican } \\
\text { catfish(Ma } \\
\text { rve Fish) }\end{array}$ & - & - & - & + & - & - & + \\
\hline
\end{tabular}

Note: PRESENT (+); ABSENT (-)

Annexure 9:-List of Mammals species recorded during the study period in studied lakes

\begin{tabular}{|c|c|c|c|c|c|c|c|c|}
\hline $\begin{array}{l}\text { Scien } \\
\text { tific Name }\end{array}$ & $\begin{array}{l}\text { Common } \\
\text { Name }\end{array}$ & $\begin{array}{c}\text { M } \\
\text { argondana } \\
\text { halli } \\
\text { Hosakere }\end{array}$ & $\underset{\text { atta }}{\text { ommagh }}$ & $\underset{\text { tta }}{\text { andema }}$ & $\begin{array}{c}\text { osakereh } \\
\text { alli }\end{array}$ & $\begin{array}{l}\text { oreke } \\
\text { re }\end{array}$ & $\begin{array}{r}\text { Su } \\
\text { bramanaya } \\
\text { pura }\end{array}$ & $\underset{\text { gala }}{\operatorname{yraman}}$ B \\
\hline dicus & ow & + & + & & - & & + & + \\
\hline $\begin{array}{r}\text { Buba } \\
\text { lusbubalis }\end{array}$ & uffalo & + & + & & - & & + & - \\
\hline $\begin{array}{ll} & \text { Ovis } \\
\text { aries } & \\
\end{array}$ & $\begin{array}{ll} & S \\
\text { heep } & \\
\end{array}$ & + & + & & - & & - & + \\
\hline $\begin{array}{l}\text { Capr } \\
\text { ahircus }\end{array}$ & oat $\quad \mathrm{G}$ & + & + & & - & & + & + \\
\hline $\begin{array}{r}\text { Herp } \\
\text { estesjavanicus }\end{array}$ & ongoose $^{\mathrm{M}}$ & + & - & & - & & + & - \\
\hline $\begin{array}{l}\text { Band } \\
\text { icotabengalen } \\
\text { sis } \\
\end{array}$ & $\begin{array}{l}\text { B } \\
\text { andicoot } \\
\text { Rat }\end{array}$ & + & + & & + & & + & + \\
\hline Funa & $\mathrm{S}$ & + & + & & + & & + & + \\
\hline
\end{tabular}




\begin{tabular}{|r|r|r|r|r|r|r|r|}
\hline $\begin{array}{l}\text { mbuluspalmar } \\
\text { um quirrel }\end{array}$ & \multicolumn{1}{l|}{} & & & & \\
\hline $\begin{array}{c}\text { Cani } \\
\text { sfamiliaris }\end{array}$ & og D & + & + & +1 & + & + \\
\hline $\begin{array}{c}\text { Ptero } \\
\text { pusgiganteus }\end{array}$ & $\begin{array}{l}\text { dian In } \\
\text { Flying fox }\end{array}$ & + & + & -1 & + & + \\
\hline
\end{tabular}

Note: PRESENT (+); ABSENT (-)

Annexure 10:-List of insect species recorded during the study period in studied lakes

\begin{tabular}{|c|c|c|c|c|c|c|c|c|}
\hline Name Common & $\begin{array}{l}\text { Name of the } \\
\text { lake/Species }\end{array}$ & $\begin{array}{l}\text { M } \\
\text { argondana } \\
\text { halli } \\
\text { Hosakere } \\
\end{array}$ & $\underset{\text { atta }}{\mathrm{K}}$ & $\begin{array}{c}\text { andema } \\
\text { tta }\end{array}$ & $\begin{array}{c}\text { Hosak } \\
\text { ere- } \\
\text { halli }\end{array}$ & $\begin{array}{l}\text { Dore } \\
\text { kere }\end{array}$ & $\begin{array}{c}\text { Subram } \\
\text { ana- } \\
\text { yapura }\end{array}$ & $\begin{array}{c}\text { Byrama } \\
\text { ngala }\end{array}$ \\
\hline $\begin{array}{l}\text { Spurthro } \\
\text { ted grass hopper }\end{array}$ & $\begin{array}{r}\text { Crytaca } \\
\text { ughtheristatarica }\end{array}$ & + & - & & & & & \\
\hline \multirow{3}{*}{$\begin{array}{l}\text { horned } \\
\text { hopper }\end{array}$} & $\begin{array}{ll} & \text { Cistoce } \\
\text { rca sp. } & \end{array}$ & - & - & & & & & \\
\hline & $\begin{array}{ll}\text { AcridaE } \\
\text { xaltata }\end{array}$ & + & - & & & & & \\
\hline & $\begin{array}{ll} & \text { Acridac } \\
\text { inerea } & \end{array}$ & - & + & & & & & \\
\hline $\begin{array}{ll} & \text { Tree } \\
\text { Cricket } & \end{array}$ & $\begin{array}{l}\text { Phaner } \\
\text { optera sp. }\end{array}$ & - & + & & & & & \\
\hline $\begin{array}{l}\text { Praying } \\
\text { mantis }\end{array}$ & $\begin{array}{l}\text { Mantis } \\
\text { religiosa }\end{array}$ & + & - & & & & & \\
\hline $\begin{array}{l}\text { Domino } \\
\text { cackroach }\end{array}$ & $\begin{array}{l}\text { Thereap } \\
\text { etiveriana }\end{array}$ & - & - & & & & & \\
\hline Termite & $\begin{array}{l}\text { Odonat } \\
\text { ermes sp. }\end{array}$ & + & + & & & & & \\
\hline $\begin{array}{r}\text { Indian } \\
\text { walking Insect }\end{array}$ & $\begin{array}{l}\text { Carausi } \\
\text { usmorosus }\end{array}$ & + & + & & & & & \\
\hline Cicada & $\begin{array}{l}\text { Platyple } \\
\text { ura sp. }\end{array}$ & - & - & & & & & \\
\hline $\begin{array}{l}\text { Leaf } \\
\text { Hopper }\end{array}$ & $\begin{array}{r}\text { Eurybra } \\
\text { chystomentosa }\end{array}$ & + & - & & & & & \\
\hline $\begin{array}{ll} & \text { Water } \\
\text { Strider } & \\
\end{array}$ & sp. $\quad$ Gerris & + & + & & & & & \\
\hline Seed bug & $\begin{array}{l}\text { Spiloste } \\
\text { thuspandurus }\end{array}$ & - & - & & & & & \\
\hline $\begin{array}{ll}\text { bug } & \text { Jewel } \\
\end{array}$ & $\begin{array}{l}\text { Chrisoc } \\
\text { orisstolli }\end{array}$ & + & - & & & & & \\
\hline $\begin{array}{l}\text { Stink } \\
\text { bug }\end{array}$ & $\begin{array}{l}\text { Ethensi } \\
\text { aacuminata }\end{array}$ & - & - & & & & & \\
\hline $\begin{array}{l}\text { Hydrom } \\
\text { etra }\end{array}$ & $\begin{array}{l}\text { Hydrom } \\
\text { etrastagnorum }\end{array}$ & - & - & & & & & \\
\hline Ranatra & $\begin{array}{ll} & \text { Nepacin } \\
\text { erea } & \\
\end{array}$ & - & - & & & & & \\
\hline a $\quad$ Mesoveli & $\begin{array}{l}\text { Mesovel } \\
\text { iamulasanti }\end{array}$ & - & - & & & & & . \\
\hline $\begin{array}{c}\text { Gold } \\
\text { cross tiger beetle }\end{array}$ & $\begin{array}{r}\text { Cincide } \\
\text { laaurofasciata }\end{array}$ & - & - & & & & & \\
\hline $\begin{array}{ll}\text { beetle } & \text { Bess } \\
\end{array}$ & $\begin{array}{r}\text { Bascilia } \\
\text { nusstoliczkae }\end{array}$ & - & - & & & & & \\
\hline $\begin{array}{l}\text { Elephant } \\
\text { Dung beetle }\end{array}$ & $\begin{array}{ll} & \text { Sisyphu } \\
\text { s sp. } & \end{array}$ & + & - & & & & & \\
\hline
\end{tabular}




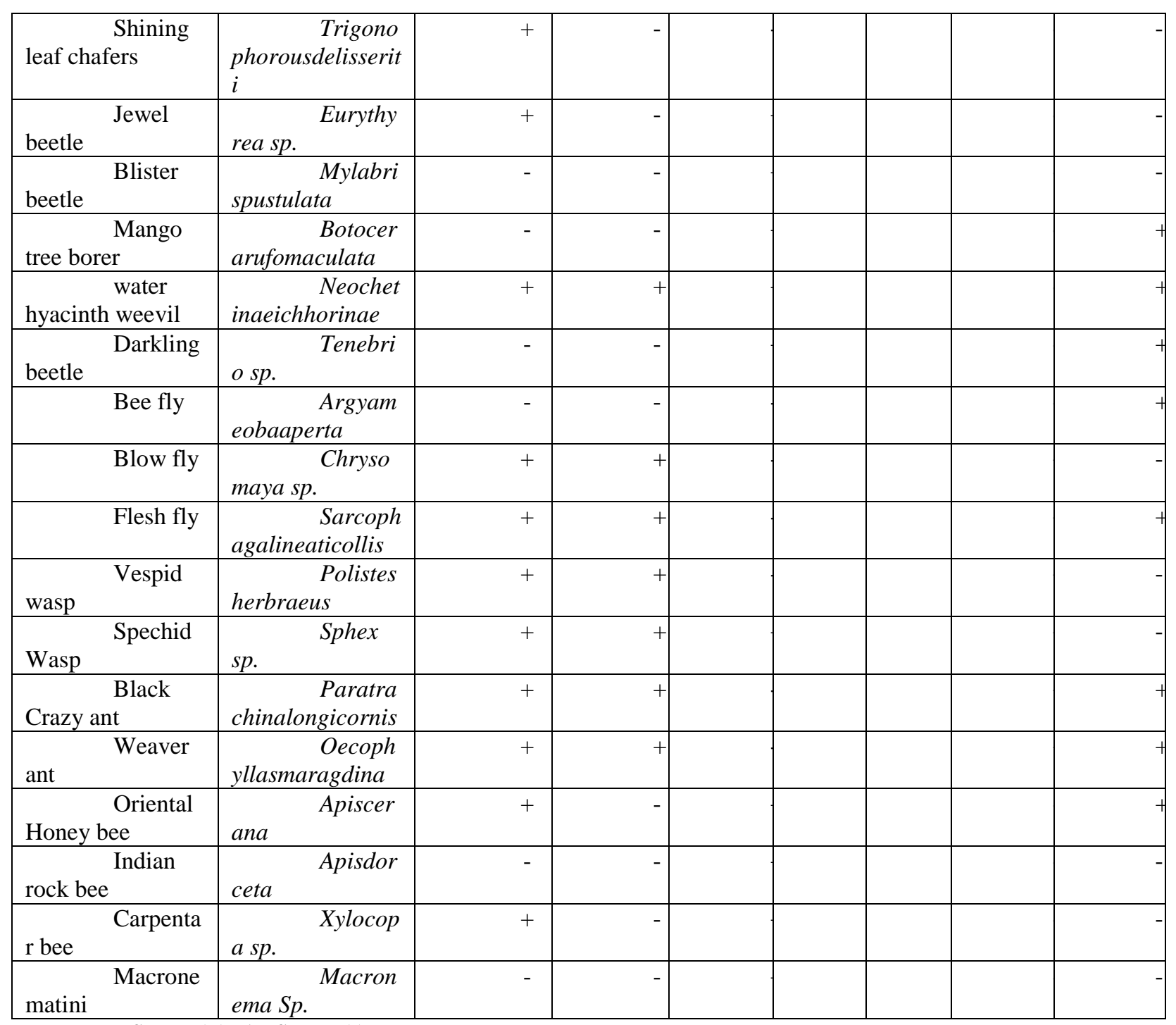

Note: PRESENT (+); ABSENT (-)

\section{References:-}

1. Bhatnagar.. C, K. Jani \& Sharma..V. (2007). Vanishing habitats of aquatic birds in the city of lakes, Udaipur: a case study. The Indian Forester. 133: 1395-1402.

2. Bookhout., T.A. (1994). Research and Management Techniques for Wildlife and Habitats, $5^{\text {th }}$ edn. Bethesda, Maryland. The Wildlife Society.

3. Donald Borror, J., \& Richard White.(1970). A Field Guide to Insects America north of Mexico. The Peterson Field Guide Series.Houghten Mifflin Company Booston Newyork.

4. Fredrick Jones Muyodi., Fredrick L., Mwanuzi., \& Raphael Kapiyo. (2011). Environmental Quality and Fish Communities in Selected Catchments of Lake Victoria. The Open Environmental Engineering Journal:54 - 65.

5. Hutchins., Dennis Thoney, A., Paul Loiselle, V., and Neil Schlager. (2003). Farmington Hills, MI: Gale Group. Grzimek's Animal Life Encyclopedia, $2^{\text {nd }}$ edition. Volume 5, Produced by Schlager Group Inc.

6. Jayaram, K.C. (1999). The Freshwater Fishes of the Indian Region, Narendra Publishing House, New Delhi.

7. Jim Erickson. (2011). Biodiversity improves water quality in streams through a division of labor. Retrieved from http://ur.umich.edu/1011/Apr11_11/2245-biodiversity-improves-water.

8. Karen Edelstein. (1999). Pond and Stream Safari: A Guide to the Ecology of Aquatic Invertebrates. 4 H Leader's Guide 147L24. A Cornell Cooperative Extension Publication:65- 91.

9. KrushnameghKunte. (2008). Butterflies of Peninsular India.Published by University Press (India) Private Limited, Hyderabad, India. 
10. Mark Huff H., Kelly Bettinger, A., Howard, L., Ferguson., Martin Brown J and Bob Altmon. (2000). A Habitat Based point Count protocol for Terrestrial Birds, Emphasizing Washington and Oregon, General Technical Report: 10 - 19.

11. MeenakshiVenkataraman. (2010). Indian Insects and Arachnids. A Concise Field Guide, $1^{\text {st }}$ edition, published by Simova Education and Research, India.

12. Meli, P., Benayas, J. M. R., Balvanera, P., and Ramos, M. M. (2014). Restoration enhances wetland biodiversity and ecosystem service supply, but results are context-dependent: a meta-analysis. PloS one, 9(4), e93507. Retrieved from <https://www.ncbi.nlm.nih.gov/pmc/articles/PMC3990551/ >

13. Mukherjee.A, Borad. C.K., and Parasharya..B.M. (2002).A study of the ecological requirements of waterfowl at manmade reservoirs in Kheda District, Gujarat, India, with a view towards conservation, management and planning. Zoos' Print J. 17: 775 - 785.

14. Nelson, E. J., and Booth, D. B. (2002). Sediment sources in an urbanizing,mixed land-use watershed. Journal of Hydrology, 264(1): 51-68.

15. Quentin Bone Richard., and Moore, H. (2008). Biology of Fishes, $3^{\text {rd }}$ ed. Taylor and Francis Group.

16. Rema Devi, K., and Indra, T.J. (2009). Check list of the native Freshwater fishes of India. Marine Biology Regional Centre, Southern Regional Centre, Zoological Survey of India, Chennai.

17. Subramanian, K.A. (2005). Dragonflies and Damselflies of Peninsular India A Field Guide. Centre for Ecological Sciences, Indian Institute of Science and Indian Academy of Sciences, Bangalore, India.

18. Subramanian, K.A. (2009). Dragonflies of India - A field Guide, Published by VigyanPrasar, department of Science and technology, Noida, India.

19.Subramanian, K.A., and Sivaramakrishnan., K.G. (2007). Aquatic Insects for Biomonitoring Freshwater Ecosystems. A Methodology Ashoka Trust for Research in Ecology and Environment (ATREE), Bangalore, India.

20. William. (2006). Ecological Census Techniques. A Handbook, Second Edition, Cambridge University Press. 